\title{
A relação entre o suicídio e o isolamento social causado pelo COVID-19
}

\author{
The connection between suicide and social isolation caused by COVID-19
}

\author{
Andressa Lays do Nascimento Cunha ${ }^{\circ}$, Camila de Macedo Rodrigues, Cristiallan Sousa Oliveira ${ }^{\circ}$, \\ Glennda Vitoria Francelino da Silva* ${ }^{\circ}$, Grasiella da Silva Santos, Ravenna Araujo Sousa ${ }^{\circ}$, Vanessa \\ Maria Pereira da Silva ${ }^{\circ}$, Bruno da Silva Gomes ${ }^{\circ}$
}

Centro de Ensino Unificado do Piauí, Teresina, Piauí, Brasil. *Autor para correspondência. E-mail: glenndahsilva@gmail.com

\begin{abstract}
Resumo: Introdução: O presente artigo visa apontar fatores relacionados a atual pandemia que contribuem para um declínio na saúde mental, sobretudo o crescente número de casos de suicídio. Revisão: O estudo foi feito por meio de revisão bibliográfica, no qual 9 artigos foram selecionados de acordo com os descritores, juntamente com 1 livro para auxiliar no desenvolvimento do estudo. Discussão: Com isso, foi possível observar que houve mudanças de comportamento visando o controle do contágio do coronavírus, em especial o isolamento social, que desencadeou uma série de agravantes do suicídio, pois, o ser humano necessita de convívio social para uma saúde mental equilibrada. Tudo isso ressalta a necessidade ao cuidado não só a doença causada pelo vírus em si, mas também com as consequências deixadas durante a pandemia a respeito da saúde mental, uma vez que, as pesquisas apresentadas apontaram que os casos de pessoas que necessitam de atendimento psicológico são maiores que o número de infectados pela COVID-19. Além disso, as pesquisas também mostram que o número só tende a crescer, mesmo após o fim da pandemia, vê-se assim que a problemática abordada deve ser tratada como uma grave questão de saúde pública. Considerações finais: Em virtude do que foi analisado, pode-se evidenciar como resultado um alto índice de mortalidade por suicídio pelos fatores supracitados, indicando a necessidade de melhoria na assistência e nos cuidados à saúde mental mediante a situação pandêmica, ampliando a possibilidade de evitar o ato suicida, fazendo com que o seu índice diminua.
\end{abstract}

Palavras-chave: COVID-19, isolamento social, saúde mental, suicídio.

\begin{abstract}
Introduction: The present paper aims to point out factors related to the current pandemic that contribute to a decline in mental health, especially the growing numbers of suicide cases. Review: The review was made by bibliographic references, which 9 articles were selected according to the descriptors, along with 1 book to assist in the development of the study. Discussion: Therewith, it was possible to observe that there have been behavioral changes aimed at controlling the contagion of the coronavirus, especially social isolation, which has triggered a series of aggravating factors of suicide, because the human being needs social interaction for a balanced mental health. All this highlights the need for care not only to the disease caused by the virus itself, but also with the consequences left during the pandemic regarding mental health, since the research presented pointed out that the cases of people who need psychological care are higher than the number of those infected by COVID-19. Besides, the research also shows that the numbers only tends to grow, even after the end of the pandemic, so we see that the problem addressed must be treated as a serious public health issue. Final considerations: As a result of what was analyzed, a high mortality rate by suicide can be evidenced as an effect of the above-mentioned factors, indicating the need for improvement in mental health assistance and care through the pandemic situation, increasing the possibility of avoiding suicide, thus reducing its rate.
\end{abstract}

Keywords: COVID-19, mental health, social isolation, suicide.

\section{Introdução}

Em dezembro de 2019, surgiu o primeiro caso do novo coronavírus na cidade de Wuhan, na China. O Coronavírus é uma família de vírus que causa infecções respiratórias podendo ser transmitido por meio de tosse ou espirro ocasionados por indivíduos infectados. No Brasil foi identificado o primeiro caso em fevereiro de 2020 na cidade de São Paulo, tendo o primeiro óbito confirmado um mês depois da sua descoberta. No mesmo mês a Organização Mundial da Saúde (OMS) decretou o COVID-19 como Pandemia induzindo ao isolamento social (OPAS/OMS). 
O distanciamento social infligiu uma mudança de comportamento na rotina da população mundial desencadeando emoções negativas que afetaram fortemente o bem-estar psicológico. Uma das consequências dessa abrupta mudança de hábitos foi o aumento dos casos de suicídio, sendo esse um ato humano de infligir a si próprio o fim da vida (Teixeira et al., 2018). Esse comportamento tem como principais motivações medo de contágio, estresse, transtornos psiquiátricos, luto e desemprego. Ademais, a demanda de saúde mental entre os profissionais da saúde, principalmente da linha de frente, intensificou-se devido à quantidade de mortes diárias (Sher, 2020b).

Vale ressaltar o impacto da última pandemia, Influenza ou Gripe Espanhola (1918-1919), na saúde mental da população. Cerca de 500 milhões pessoas foram infectadas pelo vírus e pelo menos 50 milhões morreram em todo mundo, dessa maneira, foi proposto que houvesse a diminuição do contato social (Sher, 2020b). Há evidências que mostram que durante a pandemia da gripe espanhola as mortes por suicídio aumentaram nos Estados Unidos da América. Outro ponto a ser destacado é que durante o surto da Influenza as pessoas tinham medo de serem infectadas e devido ao distanciamento social houve um declínio na saúde mental da população, o mesmo é observado na Pandemia da COVID-19 (Gunnel et al., 2020). O presente artigo tem como objetivo aprofundar os conhecimentos sobre a relação entre isolamento social causado pela pandemia do COVID-19 com o crescente número de casos de suicídios, por meio de uma revisão sistemática.

\section{Revisão}

O estudo em questão trata-se de uma revisão bibliográfica qualitativa no qual foram realizadas pesquisas na literatura sobre o tema. Os artigos encontrados estavam nos idiomas português e inglês. Para produção do estudo foram utilizadas como fonte de coleta de dados: SciElo, Literatura Latino-Americana e do Caribe em Ciências da Saúde (Lilacs) e Pubmed. Foram usados como descritores: suicídio, isolamento social, saúde mental e COVID-19. Após as pesquisas foram encontradas 657 trabalhos que abordassem o tema proposto ou que fossem semelhantes, em seguida foram pré-selecionados 100 artigos que estavam relacionados com a temática. Após uma análise, leitura de resumos e discussão foram selecionados 9 artigos e 1 livro para a fabricação desse estudo.

\section{Resultados e discussão}

Quadro 1. Artigos e livro utilizados na produção dessa revisão bibliográfica.

\begin{tabular}{|c|c|c|}
\hline Autor(es) & Título & Resumo \\
\hline John et al. (2020) & $\begin{array}{l}\text { Tendências em suicídio } \\
\text { durante a pandemia } \\
\text { COVID-19 }\end{array}$ & $\begin{array}{l}\text { Como muitos países enfrentam as novas restrições para ficar em casa para } \\
\text { conter a disseminação do covid-19, há preocupações que taxas de suicídio } \\
\text { possam aumentar - ou já tenham aumentado. Muitos fatores sustentam } \\
\text { essas preocupações, incluindo a deterioração da saúde mental da população, } \\
\text { uma maior prevalência de pensamentos e comportamentos relatados de } \\
\text { automutilação entre pessoas com covid-19, problemas para acessar } \\
\text { programas de ajuda a saúde mental, e evidencias que em outras epidemias } \\
\text { como a SARS (2003) foram associadas com um crescimento de mortes por } \\
\text { suicídio. }\end{array}$ \\
\hline $\begin{array}{l}\text { Gunnell et al. } \\
\text { (2020) }\end{array}$ & $\begin{array}{l}\text { Risco de suicídio e } \\
\text { prevenção durante a } \\
\text { pandemia COVID-19 }\end{array}$ & $\begin{array}{l}\text { Os efeitos sobre a saúde mental na pandemia do COVID-19 podem ser } \\
\text { profundos e a longo prazo, portanto a prevenção do suicídio precisa de } \\
\text { consideração urgente. O desemprego, isolamento social, aprisionamento e } \\
\text { solidão, acesso aos meios de comunicação com reportagens irresponsáveis } \\
\text { são fatores de riscos para o suicídio, podendo variar dependendo do } \\
\text { contexto sociocultural e saúde pública de cada país. Disponibilizar recursos } \\
\text { onlines gratuitos baseados em evidências e intervenções pode beneficiar a } \\
\text { saúde mental da população. }\end{array}$ \\
\hline Durkheim (2000) & O Suicídio & $\begin{array}{c}\text { A obra "O suicídio" de Durkheim escrita em } 1897 \text { diz respeito a um assunto } \\
\text { considerado psicológico na condição de fenômeno social, a fim de provar } \\
\text { que o suicídio é um fato social, por meio de coerção exterior e independente } \\
\text { do indivíduo, inserida na sociedade. }\end{array}$ \\
\hline Lima (2020) & $\begin{array}{l}\text { Distanciamento e } \\
\text { isolamento sociais pela } \\
\text { Covid-19 no Brasil: } \\
\text { impactos na saúde mental }\end{array}$ & $\begin{array}{l}\text { A pandemia de coronavírus tem atravessado todo o tecido social, não } \\
\text { poupando praticamente nenhuma área da vida coletiva ou individual, com } \\
\text { repercussões na esfera da saúde mental. }\end{array}$ \\
\hline $\begin{array}{c}\text { Pereira et al. } \\
\text { (2018) }\end{array}$ & $\begin{array}{l}\text { Fatores de risco e proteção } \\
\text { para tentativa de suicídio }\end{array}$ & $\begin{array}{l}\text { O impacto do isolamento social foi alto, e com isso o veio o surgimento do } \\
\text { medo de serem infectados juntamente com a preocupação com a família e }\end{array}$ \\
\hline
\end{tabular}




\begin{tabular}{|c|c|c|}
\hline & na adultez emergente. & $\begin{array}{l}\text { amigos. A tristeza em decorrência da pandemia deteriorou a saúde mental. } \\
\text { As pesquisas feitas mostram muitas dificuldades com a população no } \\
\text { quesito psicológico. }\end{array}$ \\
\hline Sher (2020a) & $\begin{array}{l}\text { Are COVID-19 survivors at } \\
\text { increased risk for suicide? }\end{array}$ & $\begin{array}{l}\text { O surto da doença coronavírus (COVID-19) surgiu pela primeira vez na } \\
\text { China no final do ano passado. A epidemia de COVID-19 se espalhou para } \\
\text { todos os continentes. Milhões de pessoas adoeceram com COVID-19. Nesta } \\
\text { carta ao editor, sugiro que os sobreviventes do COVID-19, especialmente os } \\
\text { indivíduos com COVID-19 grave, apresentam risco aumentado de suicídio. } \\
\text { O suicídio entre indivíduos que tiveram COVID-19 pode estar relacionado a } \\
\text { fatores psicológicos e neurobiológicos. }\end{array}$ \\
\hline Sher (2020b) & $\begin{array}{l}\text { Impact of the covid-19 } \\
\text { pandemic on suicide rates }\end{array}$ & $\begin{array}{l}\text { Várias linhas de evidência indicam que a pandemia da doença coronavirus } \\
2019 \text { deixará sequelas psicológicas que provavelmente persistirão por meses } \\
\text { e anos. }\end{array}$ \\
\hline Sher (2019) & $\begin{array}{l}\text { Resilience as a focus of } \\
\text { suicide research and } \\
\text { prevention }\end{array}$ & $\begin{array}{c}\text { O suicídio é um grande problema médico e social. Décadas de pesquisas } \\
\text { sobre suicídio se concentraram principalmente nos fatores de risco para } \\
\text { comportamento suicida, ao mesmo tempo em que negligenciam os fatores } \\
\text { de proteção, como a resiliência, que podem ajudar a resolver esse } \\
\text { importante problema de saúde pública. }\end{array}$ \\
\hline $\begin{array}{l}\text { Soccol \& Silveira } \\
\quad(2020)\end{array}$ & $\begin{array}{l}\text { Impactos do } \\
\text { distanciamento social na } \\
\text { saúde mental: estratégias } \\
\text { para a prevenção do } \\
\text { suicídio } \\
\end{array}$ & $\begin{array}{c}\text { O distanciamento intensificou ainda mais os sintomas de depressão, } \\
\text { ansiedade e sentimentos de medo e solidão das pessoas, principalmente } \\
\text { daquelas que já possuíam histórico de transtorno mental, levando-as a } \\
\text { tentativa e ao suicídio. }\end{array}$ \\
\hline $\begin{array}{l}\text { Teixeira et al. } \\
\text { (2018) }\end{array}$ & $\begin{array}{l}\text { O suicídio como questão de } \\
\text { saúde pública }\end{array}$ & $\begin{array}{l}\text { O suicídio caracteriza-se como um fenômeno complexo, emergente e } \\
\text { multidimensional, ligado a contextos sociais, históricos e econômico. } \\
\text { Conhecer essas questões para trabalhar os fatores preventivos, criação de } \\
\text { programas, estruturação das polícias públicas, onde esse problema de saúde } \\
\text { pública possa ter um percurso de ação mais eficiente. } \\
\text { O suicídio como questão de saúde pública }\end{array}$ \\
\hline
\end{tabular}

Desde o surgimento do coronavírus tem se procurado condutas que diminuam o seu avanço e contágio, como por exemplo, lavar as mãos frequentemente, uso de máscaras, uso de álcool em gel e principalmente isolamento social (Lima, 2020). Émile Durkheim em seu famoso livro "O Suicídio" diz que os indivíduos necessitam de uma conexão social, quando há a diminuição ou falta desse contato com ambiente coletivo, pode haver o aumento das taxas de suicídio. Os prováveis efeitos adversos da pandemia em pessoas com transtorno mental e na saúde mental da população em geral podem ser exacerbados pelo medo, isolamento subjetivo e distanciamento físico. O risco de suicídio pode aumentar devido ao estigma em relação a indivíduos com COVID-19 e suas famílias (John et al., 2020). Além disso, a COVID-19 desencadeia o medo da morte, sentimentos de desamparo, abandono, insegurança, angústia, tédio e entre outros (Soccol \& Silveira, 2020).

Apesar da conexão social continuar estabelecida através das mídias, essas redes também são responsáveis pelo o aumento da ansiedade, estresse, medo de contágio devido ao excesso e sensacionalismo de informações (Sher, 2020b). Ainda a respeito do impacto da mídia na saúde mental, Gunnel et al. (2020) diz que as reportagens irresponsáveis e excesso de divulgação de casos de suicídio podem gerar medo e aumentar os casos de pessoas que põe fim na própria vida, principalmente quando a população está sem contato com o ambiente social.

Ademais, pesquisas apontam que a sensação subjetiva de estar sozinho está relacionada a ideação e comportamento suicida. Há uma grande probabilidade de que os sobreviventes de COVID-19, especialmente aqueles que tiveram sintomas graves ou passaram pela UTI, estejam em risco elevado de suicídio já que os pacientes podem desenvolver ansiedade, depressão, estresse pós-traumático. Vale ressaltar que a depressão é um fator de risco para o suicídio, sendo ela a maior responsável pelas mortes causadas pelo ato (Sher, 2020b). Além disso, Sher (2020a) acredita que perceber que está com coronavírus pode gerar um impacto psicológico grave, principalmente devido isolamento social e o medo de infectar outras pessoas. Deve-se ressaltar que muitos pacientes com COVID-19 e que estão em recuperação enfrentam a perda de emprego e as dificuldades financeiras, este fator psicossocial contribui para o suicídio (Sher, 2019).

Partindo disso, pode-se observar que diversos fatores contribuíram para um agravamento no quesito saúde, pois comumente, o número de infectados pela doença é menor do que o número de pessoas que necessitam de tratamento psicológico (Lima, 2020), sendo o Brasil, o $8^{\circ}$ país no mundo com o maior número casos de suicídio e tendo no último ano uma piora abrupta na saúde mental, mostrando a necessidade do 
foco à assistência psicológica e tornando como uma demanda de saúde pública (Teixeira et al., 2018). De acordo com Ann John et al. (2020), muitos fatores sustentam essas preocupações acerca do aumento das taxas de suicídio como a deterioração da saúde mental da população, maior prevalência de pensamentos e comportamentos de automutilação, dificuldade para o acesso de programas de saúde mental.

A pandemia causará sofrimento e deixará muitas pessoas vulneráveis a transtornos mentais e comportamentos suicidas, assim, as consequências da pandemia sob a saúde mental da população tende a durar e causar picos maiores após o seu fim (Gunnel et al., 2020). Deve-se salientar que as tentativas de suicídio são consideradas situações evitáveis, dessa maneira, é importante estabelecer estratégias para a prevenção do suicídio (Soccol \& Silveira, 2020). Como medida de segurança para evitar o contágio, as clinicas de psicologia tiveram que parar com o atendimento presencial, dessa forma, o Conselho Federal de Psicologia (CFP), registrou um aumento de mais de 50 mil solicitações para registro do atendimento psicoterapêutico online (CFP, 2020). Portanto, é importante estabelecer estratégias de prevenção ao suicídio durante a pandemia como a atuação de equipes multiprofissionais da saúde mental, manter a comunicação com os usuários e familiares das Redes de Atenção Psicossocial (RAPS), atendimento psicológico online. É necessário que, principalmente, durante o período pandêmico as pessoas se sintam acolhidas, amparadas, escutadas. Ademais, as redes sociais e mídias servem como unificadoras de contato, fortalecendo a ligação entre familiares e amigos, o sentimento de pertencimento, desse modo, as taxas de suicídio diminuem (Soccol \& Silveira, 2020).

\section{Considerações finais}

Portanto, de acordo com o exposto, fatores como ansiedade, depressão, estresse, luto, medo de contágio implantados pelo isolamento social tem contribuído para o aumento de casos de suicídio além do excesso e/ou sensacionalismo de informações a respeita da COVID-19. Ademais, é necessário que haja um cuidado maior com as vítimas de coronavírus, especialmente as que tiveram sintomas mais graves ou estiveram na UTI, pois elas possuem maior tendência a desenvolver ansiedade, depressão, estresse pós-traumático além do medo de contaminar outras pessoas. Fatores psicossociais como o desemprego e dificuldades financeiras podem influenciar os indivíduos a cometerem suicídio.

Por fim, o impacto do isolamento social provocado pela disseminação do COVID-19 em relação ao suicídio se torna uma questão de saúde pública no Brasil devido o país ser o $8^{\circ}$ no mundo com mais casos de pessoas que põe fim na própria vida. Desse modo, se faz necessária medidas de prevenção ao suicídio durante a pandemia, como manter as relações sociais através das mídias que são unificadoras, manter o contato com os usuários e familiares dos RAPS, fazer com que as pessoas se sintam acolhidas e ouvidas.

\section{Referências}

Durkheim, E. 2000. O Suicídio (5a ed.). São Paulo, SP: WMF Martins Fontes.

Gunnell, D., Appleby, L., Arensman, E., Hawton, K., John, A., Kapur, N., Khan, M., O'Connor, R. C. \& Pirkis, J. 2020. Suicide risk and prevention during the COVID-19 pandemic. The Lancet Psychiatry, 7(6), 468-471.

John, A., Pirkis, J., Gunnell, D., Appleby, L., \& Morrissey, J. 2020. Trends in suicide during the covid-19 pandemic. The BMJ, 371, $\mathrm{m} 4352$.

Lima, R. C. 2020. Distanciamento e isolamento sociais pela Covid-19 no Brasil: impactos na saúde mental. Physis: Revista de Saúde Coletiva, 30(2), e300214, 1-10.

OPAS/OMS. Organização Pan-Americana da Saúde/Organização Mundial da Saúde. Folha informativa sobre COVID-19. Disponível em: https://www.paho.org/pt/covid19. Acesso em: 28 de abril de 2021.

Pereira, A. S., Wilhelm, A. R., Koller, S. H., \& Almeida, R. M. M. 2018. Fatores de risco e proteção para tentativa de suicídio na adultez. Ciência \& Saúde Coletiva, 23(11), 3767-3777.

Sher, L. 2019. Resilience as a focus of suicide research and prevention. Acta Psychiatrica Scandinavica, 140(2), 169-180.

Sher, L. 2020a. Are COVID-19 survivors at increased risk for suicide? Acta Neuropsychiatrica, 32(5), 270.

Sher, L. 2020b. The impact of the COVID-19 pandemic on suicide rates. QJM: Monthly Journal of the Association of Physicians, 113(10), 707-710. 
Soccol, K. L. S., \& Silveira, A. 2020. Impactos do distanciamento social na saúde mental: estratégias para a prevenção do suicídio. Journal of Nursing and Health, 10, e20104033.

Teixeira, S. M. O., Souza, L. E. C., \& Viana, L. M. M. 2018. O suicídio como questão de saúde pública. Revista Brasileira em Promoção da Saúde, 31(3), 1-3.

\section{Minicurrículo}

Andressa Lays do Nascimento Cunha. Acadêmica de Psicologia no Centro de Ensino Unificado do Piauí (CEUPI), Teresina- PI, Brasil.

Camila de Macedo Rodrigues. Acadêmica de Psicologia no Centro de Ensino Unificado do Piauí (CEUPI), Teresina- PI, Brasil.

Cristiallan Sousa Oliveira. Acadêmico de Psicologia no Centro de Ensino Unificado do Piauí (CEUPI), Teresina- PI, Brasil.

Glennda Vitoria Francelino da Silva. Acadêmica de Psicologia no Centro de Ensino Unificado do Piauí (CEUPI), Teresina- PI, Brasil.

Grasiella da Silva Santos. Acadêmica de Psicologia no Centro de Ensino Unificado do Piauí (CEUPI), Teresina- PI, Brasil.

Ravenna Araujo Sousa. Acadêmica de Psicologia no Centro de Ensino Unificado do Piauí (CEUPI), Teresina- PI, Brasil.

Vanessa Maria Pereira da Silva. Acadêmica de Psicologia no Centro de Ensino Unificado do Piauí (CEUPI), Teresina- PI, Brasil.

Bruno da Silva Gomes. Bacharel em Ciências Biológicas, mestre em Farmacologia, Doutor em Biotecnologia, Professor no Centro de Ensino Unificado do Piauí (CEUPI), Teresina-PI, Brasil.

Como citar: Cunha, A.L.N., Rodrigues, C.M., Oliveira, C.S., Silva, G.V.F., Santos, G.S., Sousa, R.A., Silva, V.M.P., \& Gomes, B.S. 2021. A relação entre o suicídio e o isolamento social causado pelo Covid-19. Pubsaúde, 6, a212. DOI: https://dx.doi.org/10.31533/pubsaude6.a212

Recebido: 9 jun. 2021.

Revisado e aceito: 24 jun. 2021.

Conflito de interesse: os autores declaram, em relação aos produtos e companhias descritos nesse artigo, não ter interesses associativos, comerciais, de propriedade ou financeiros que representem conflito de interesse.

Licenciamento: Este artigo é publicado na modalidade Acesso Aberto sob a licença Creative Commons Atribuição 4.0 (CC-BY 4.0). 